\title{
Die sechste Welle der Verhaltenstherapie: Biografie und Konstitution
}

\author{
Michael Linden \\ Rehabilitationszentrum Seehof der Deutschen Rentenversicherung, Verhaltenstherapie/Psychosomatik, Teltow, Deutschland
}

Zur Bestimmung dessen, was Verhaltenstherapie (VT) ist, sind in diesem Heft 2 Beiträge von grundsätzlicher Bedeutung: Im Beitrag von Ulrich Schweiger zum Verfahren «Verhaltenstherapie» wird eine Definition von VT in Anlehnung an die Formalvorgaben des Wissenschaftlichen Beirats Psychotherapie gegeben. In Ergänzung dazu ist die Übersichtsarbeit von Ulrike Ehlert und Pearl La Marca-Ghaemmaghami zur Exploration von pränatalem Stress für die Therapieindikation von besonderem Interesse, da darin gezeigt wird, wie sich VT theoretisch und therapeutisch weiterentwickelt, ohne dass deswegen das Verfahren «Verhaltenstherapie» infrage gestellt werden muss.

Die theoretischen Grundlagen wie auch das therapeutische Arsenal der VT haben sich seit ihrem Beginn in den 1950er Jahren etwa im 10-Jahres-Rhythmus um wesentliche Aspekte erweitert. In diesem Zusammenhang hat sich der Begriff der «Wellen» etabliert. Am Anfang stand die klassische Lerntheorie, die übersetzt wurde in die systematische Desensibilisierung nach Joseph Wolpe [1973]. Dieses Verfahren wurde bei der Behandlung von Angstreaktionen angewendet, die als Ausdruck einer Konditionierung verstanden wurden. Mit Rückgriff auf die operante Konditionierung wurde dann auch beispielsweise die Token Economy entwickelt, das heißt die Veränderung von Verstärkern zur systematischen Verhaltensänderung.

In den 1960er Jahren wurden dann als zweite Welle Konzepte der Sozialpsychologie integriert. Dabei wurden Verstärker in Abhängigkeit zu den sozialen Kompetenzen eines Individuums verstanden. Beispielhaft war die Entwicklung des Trainings der sozialen Kompetenz [Ullrich und de Muynck, 1973].

In den 1970er Jahren kamen in der dritten Welle die kognitiven Modelle hinzu, beispielsweise durch Ellis [1997] oder Beck [1975]: Obwohl Menschen eigentlich die Kompetenz haben, bestimmte Dinge zu tun, so sind sie dennoch wegen hinderlicher Kognitionen dazu nicht in der Lage.

Als vierte Welle fand dann in den 1980er Jahren Berück- sichtigung, dass Kognitionen zwar Einfluss auf Emotionen haben, diese aber auch unabhängig davon Kognitionen und Verhalten beeinflussen, sodass beispielsweise in der Dialektisch-Behavioralen Therapie (DBT) nach Linehan [1987] Strategien entwickelt wurden, die auf die direkte Beeinflussung von Emotionen abzielten.

Die fünfte Welle kam in den 1990er Jahren mit sogenannten störungsspezifischen Therapieprogrammen. Diese Entwicklung war bedingt durch die Etablierung der Psychotherapie als Krankenbehandlungsmethode, wofür der störungsspezifische Wirksamkeitsnachweis eine Voraussetzung war.

Im vergangenen Jahrzehnt kam nun als sechste Welle die Berücksichtigung der individuellen Konstitution als weiteres Element hinzu. Dies betrifft genetische Aspekte, pränatale Prägungen oder frühkindliche Erfahrungen einschließlich Traumata, die sich sowohl in individuellen biologischen Reaktionsbereitschaften (z.B. Stressreagibilität) wie auch psychologischen Charakteristika (z.B. kognitiven Schemata) niederschlagen. Am Beispiel der Auswirkungen von biologischem und/oder psychologischem pränatalen Stress geben Ehlert und La Marca-Ghaemmaghami eine Übersicht, welche überdauernden Änderungen in der Reaktionsbereitschaft der Betroffenen daraus erwachsen können. Der Mensch ist ein Wesen, das von der Zeugung an durch Umwelteinflüsse konstitutionell geprägt wird, von der Größe der Nebennierenrinde bis zu kognitiven Schemata. Wie in der Arbeit von Ehlert und La Marca-Ghaemmaghami angesprochen wird, sind konstitutionelle Prägungen eines Menschen in der Regel irreversibel. Auch durch noch so viele Stunden an Psychotherapie lässt sich nach derzeitigem Wissen keine ätiologisch orientierte Veränderung herbeiführen. Als therapeutischer Ansatz bleibt nur ein kompensatorisches Vorgehen, wie es auch bereits in der vierten VT-Welle erkannt wurde, weshalb man von «dialektischer»VT sprach; d.h. man behandelt Reaktionslagen, die eigentlich nicht veränderbar sind. VT kann auch dann positive Wirkungen haben, wenn eine kausale Therapie letztlich nicht möglich ist.

\section{KARGER \\ Fax +497614520714

\section{(C) 2014 S. Karger GmbH, Freiburg}

$1016-6262 / 14 / 0244-0241 \$ 39.50 / 0$
Prof. Dr. Michael Linden

Rehabilitationszentrum Seehof der Deutschen Rentenversicherung

Verhaltenstherapie/Psychosomatik

Lichtenfelder Allee 55, 14513 Teltow, Deutschland

michael.linden@charite.de 
Allerdings muss vor einigen Missverständnissen gewarnt werden. Es wäre falsch zu glauben, dass man über eine retrospektive biografische Analyse Rückschlüsse auf den jetzigen Zustand des Patienten ableiten bzw. mit Blick auf frühe Erfahrungen die jetzige Therapie steuern könnte. Die Tatsache, dass es in einer Familie eine genetische Belastung oder während der Schwangerschaft oder frühen Kindheit Probleme oder sogar Traumata gab, heißt nicht, dass dies beim Patienten zu irgendeiner Konsequenz geführt haben muss und schon gar nicht, dass sich daraus retrospektiv orientierte und ursachenbezogene therapeutische Ansätze ergeben.

Das Wissen um konstitutionelle Unterschiede zwischen Patienten kann stattdessen die Mikroverhaltensanalyse leiten. Es kann auf der Basis von Befunden, wie sie beispielsweise von Ehlert und La Marca-Ghaemmaghami berichtet werden, gezielt und theoriegeleitet geprüft werden, ob die konstitutionelle Reaktionsbereitschaft im Sinne der Organismusvariable im konkreten Fall Besonderheiten aufweist. Während die VT klassischerweise davon ausging, dass Angststörungen in uniformer Weise durch Exposition zu behandeln sind, folgt aus der Berücksichtigung der Konstitution, dass es Patienten mit unterschiedlicher Reaktionsbereitschaft auf Stressoren oder Lebenssituationen gibt und daher die Therapie entsprechend unterschiedlich zu gestalten ist. Abhängig von der individuellen Konditionierbarkeit und Stressreagibilität darf beispielsweise nicht jeder Patient mit Expositionsmethoden behandelt werden. In der Verhaltensanalyse ist im Hier und Jetzt die konstitutionelle Reaktionsbereitschaft der Patienten - seien es biologische oder psychologische Faktoren - stärker ins Auge zu fassen.

Die Berücksichtigung konstitutioneller und damit auch biografischer Aspekte macht aus der VT dennoch keine psychodynamisch-integrative Therapie, wie gelegentlich zu lesen ist. Das Kernstück der VT ist und bleibt weiterhin die Verhaltensanalyse. Als VT kann jede Therapie verstanden werden, die auf einer Verhaltensanalyse in Anlehnung an das sogenannte SORK-Schema aufbaut. Die sechste Welle vertieft das Verständnis insbesondere der Organismusvariablen, ändert aber nichts am Kern des Verfahrens «Verhaltenstherapie».

\section{Literatur}

Beck AT: Cognitive Therapy and the Emotional Disorders. Madison, Intl Universities Press, 1975.

Ellis A: Grundlagen und Methoden der rational-emotiven Verhaltenstherapie. München, Pfeiffer, 1997. nehan MM: Dialectical Behavior Therapy for Borderline Personality Disorder: Theory and Method. Bull Menninger Clin 1987;51:261-276.
Ullrich R, de Muynck R: Assertiveness-Training-Programm (ATP); in Brengelmann J, Tunner W (eds): Behavior Therapy - Verhaltenstherapie. München, Urban und Schwarzenberg, 1973.

Wolpe J: The Practice of Behavior Therapy, ed 2. Oxford, Pergamon, 1973. 